\title{
Cicloergômetro de baixo custo com tecnologia interativa e monitoramento cardíaco para Reabilitação Pós-COVID19*
}

\author{
Anielle Martins de \\ Azevedo $^{\dagger}$ \\ Dpto. de Eng. Biomédica, \\ UFPE. Recife, PE, Brasil. \\ anielle.azevedo@ufpe.br
}

\author{
Thiago B. G. \\ Lafayette \\ Dpto. de Eng. Biomédica, \\ UFPE. Recife, PE, Brasil. \\ thiago.buarque@ufpe.br
}

\author{
Danillo Rodrigues \\ Aguiar \\ Dpto. de Eng. Biomédica, \\ UFPE. Recife, PE, Brasil. \\ danillo.aguiar@ufpe.br
}

\author{
Alana Elza Fontes da \\ Gama \\ Dpto. de Eng. Biomédica, \\ UFPE. Recife, PE, Brasil. \\ alana.elza@ufpe.br
}

\begin{abstract}
Resumo
A Covid-19 é uma doença grave que causa limitações físicas e funcionais. A reabilitação desses pacientes é fundamental, mas a adesão é pequena. Devido a isso, esse trabalho tem a proposta de desenvolver um cicloergômetro eletrônico de baixo custo associado a um aplicativo interativo com monitoramento cardiorrespiratório para melhorar a adesão dos pacientes à terapia.
\end{abstract}

\section{CCS CONCEPTS}

-Applied computing • Human-centered computing

\section{PALAVRAS CHAVES}

Cicloergômetro; Covid-19; Monitoramento cardíaco; biofeedback

\section{Introdução}

A Covid-19 é uma doença grave que afeta vários sistemas e pode resultar em morte. Sequelas a longo prazo são desconhecidas, mas as evidências de surtos anteriores demonstram comprometimento da função pulmonar, física e redução na qualidade de vida. ${ }^{(1}$ Grande parte dos pacientes afetados pela COVID-19 pode desenvolver uma síndrome pós viral grave denominada de "Síndrome Pós-Covid19", um estado de fadiga crônica. ${ }^{2)}$

O cicloergômetro é uma bicicleta estacionária que realiza rotações cíclicas. O uso do aparelho é frequente no ambiente ambulatorial, pois surgiu como uma alternativa para facilitar e potencializar a reabilitação cardiorrespiratória. ${ }^{(46)}$

As tecnologias interativas com feedback visual estão sendo utilizadas cada vez mais na área de saúde. Esses mecanismos ajudam na adesão da tarefa, no desenvolvimento da aprendizagem e no desempenho durante a terapia. ${ }^{(7-9)}$ Essas novas alternativas em saúde mostram uma solução para fisioterapia: melhorar a adesão ao tratamento, analisar os resultados e evolução dos pacientes. (10.11) Diante desse contexto, o objetivo desse trabalho é desenvolver um cicloergômetro eletrônico associado a um aplicativo com tecnologia interativa e feedback visual, mais sensor de monitoramento cardíaco e de oxigênio para melhorar a adesão dos pacientes à terapia.

\section{Motivação}

Os pacientes pós-covid19 apresentam disfunções sistêmicas como fraqueza muscular, miopatias, limitações respiratórias, físicas e funcionais, impactando diretamente na qualidade de vida. As complicações Pós-Covid19, provavelmente causarão uma pressão substancial na saúde e na assistência social nos próximos anos. $(12,13)$

O exercício aeróbio é fortemente indicado para reabilitação cardiorrespiratória ${ }^{(14)}$ Apesar de seus benefícios para melhora física, da funcionalidade e qualidade de vida, a adesão ainda é pequena. A baixa adesão pode diminuir os benefícios clínicos e gerar prejuízos econômicos para a saúde. Por essas razões, é necessário formular programas de reabilitação para esses pacientes, buscando por estratégias em inovação e desenvolvimentos tecnológicos para dinamizar a reabilitação e melhorar a adesão dos indivíduos ao tratamento. ${ }^{(15,16)}$

\section{Solução Proposta}

Esse trabalho objetiva desenvolver um cicloergômetro eletrônico com tecnologia interativa, monitoramento cardiorrespiratório e feedback visual via aplicativo para smartphones, para reabilitação de pacientes pós-Covid19 no ambiente hospitalar. A tecnologia proposta pode melhorar a adesão dos pacientes ao tratamento através do estímulo visual proposto no aplicativo. Além disso, a ferramenta poderá permitir a avaliação e análise do exercício, acompanhando as atividades no celular do profissional ao longo do processo de reabilitação.

O desenvolvimento do dispositivo mecânico, seguiu um protótipo de cicloergômetro eletrônico baseado no modelo descrito no artigo da Revista UNILUS Ensino e Pesquisa. (17) Apesar de seguir o modelo referenciado, a diferença da solução proposta está nos componentes eletrônicos, monitoramento, e comunicação com um aplicativo, que será composto por um sistema de tecnologia interativa. $\mathrm{O}$ desenvolvimento do aplicativo seguiu os passos da metodologia de inovação de listagem de requisitos e design thinking, incluindo as etapas ideação, prototipagem, validação e implementação. (18) Foi desenvolvido um protótipo de baixa fidelidade para ser validado com os profissionais da área. Ao fim, será realizada avaliação da técnica proposta. Todo o desenvolvimento do trabalho conta com o apoio de fisioterapeutas.

\section{Resultados Preliminares}

O protótipo do cicloergômetro produzido ${ }^{(18)}$ e seus componentes podem ser visualizados na figura 1 . Os resultados dos testes de alcance do sinal mostraram-se positivos em quase todas as distâncias estabelecidas. $\mathrm{O}$ parâmetro de perda de pacote 
apresentou valor nulo ( $0 \%$ de perda) nas distâncias de $1 \mathrm{~m}$ a $10 \mathrm{~m}$, nos valores acima de $10 \mathrm{~m}$ começou a ocorrer falha na comunicação do pareamento entre o módulo HC-06 e o aparelho receptor, resultando em $100 \%$ de perda dos dados. Os valores aferidos apresentaram como obstáculos paredes de alvenaria de $170 \mathrm{~mm}$ de espessura. Os testes de $10 \mathrm{~m}$ foram realizados com a presença de três paredes. O cálculo de potência através do resistor shunt indicou um consumo máximo do equipamento próximo de 1,3W. Essa potência poderia ser suprida por uma bateria de litium comercial de $2200 \mathrm{mAh}$ por um período de atividade de $3 \mathrm{~h}$. Todo o equipamento cumpriu com o esperado, sendo um desenvolvimento de baixo custo.

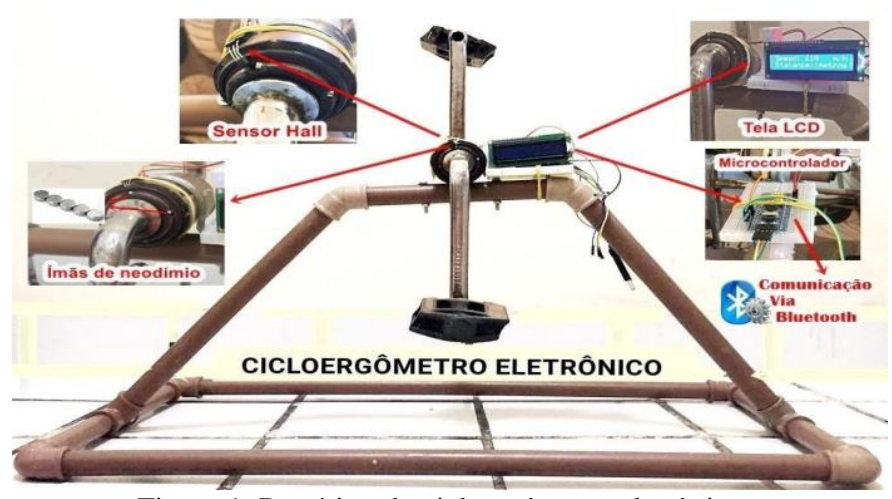

Figure 1: Protótipo do cicloergômetro eletrônico.

O cicloergômetro eletrônico também foi equipado com um sensor bluetooth, que fará a comunicação das informações do exercício (pedalada) e do monitoramento dos sinais cardiorrespiratórios diretamente para o celular. A interface inicial do aplicativo proposta pode ser vista no vídeo ${ }^{1}$. O aplicativo foi dividido em algumas etapas: A avaliação do paciente com parâmetros importantes para terapia e programação da terapia, onde o terapeuta ajusta limites de frequência cardíaca (FC) e saturação de oxigênio (SapO2) a serem atingidas pelo paciente. Durante a terapia, o paciente tem contato com a tecnologia interativa e o feedback através de um vídeo que simula locais propícios a pedaladas (podendo ser escolhido pelo paciente, exemplo: parques, estradas, campos, etc.). A velocidade do vídeo é controlada de acordo com a pedalada do usuário e de acordo com seu empenho na terapia (alto ou baixo), o paciente interage com a tela recebendo mensagens de estímulo para ir mais rápido ou devagar. Se o paciente precisa acelerar para atingir a FC programada, ele recebe mensagens de estímulo para aumentar o ritmo e se ele precisa desacelerar por ter atingido a FC máxima, recebe mensagens incentivadoras para ir mais devagar, o mesmo para os limites de SapoO2. A proposta é que no fim, um relatório da terapia seja gerado para o profissional com os valores alcançados pelo paciente. O monitoramento da FC e SapO2 será realizado através de um sensor MAX30100 e esses dados serão transferidos para o aplicativo via wireless, essa etapa do trabalho está em desenvolvimento.

\section{Conclusão}

A partir da condução desta pesquisa, prevê-se uma contribuição importante para solucionar problemas em saúde com o auxílio da tecnologia interativa. O cicloergômetro tem como seu diferencial o uso de componentes eletrônicos e comunicação com dispositivos mobile, permitindo desenvolvimento de soluções interativas. Isso nos leva a acreditar no seu potencial para melhorar a adesão e possibilitar diferentes aplicações. Para as etapas seguintes, faz-se necessário a submissão ao comitê de ética e a realização de testes clínicos.

\section{AGRADECIMENTOS}

Nossos agradecimentos à Universidade Federal de Pernambuco e ao Grupo de pesquisa de Eng. da Reabilitação.

\section{REFERÊNCIAS}

1. Barker-Davies RM, O'Sullivan O, Senaratne KPP, Baker P, Cranley M, DharmDatta S, et al. The Stanford Hall consensus statement for post-COVID-19 rehabilitation. Br J Sports Med. 2020;54(16):949-59.

2. Carruthers BM, Van de Sande MI, De Meirleir KL, Klimas NG, Broderick G, Mitchell T, et al. Myalgic encephalomyelitis: International Consensus Criteria. J Intern Med. 2011;270(4):327-38.

3. Greenleaf JE, Bernauer EM, Ertl AC, Bulbulian R, Bond M. Isokinetic strength and endurance during 30-day $6^{\circ}$ head-down bed rest with isotonic and isokinetic exercise training. Aviat Sp Environ Med. 1994;

4. Fan E, Cheek F, Chlan L, Gosselink R, Hart N, Herridge MS, et al. An official american thoracic society clinical practice guideline: The diagnosis of intensive care unit-acquired weakness in adults. Am J Respir Crit Care Med. 2014;190(12): 1437-46.

5. Needham DM, Truong AD, Fan E. Technology to enhance physical rehabilitation of critically ill patients. Crit Care Med. 2009;37(SUPPL. 10).

6. Godoy J. Biofeedback y Deportes: potenciales líneas de actuación. Eur J Hum Mov. 1995;

7. Sanderson DJ, Cavanagh PR. Use of augmented feedback for the modification of the pedaling mechanics of cyclists. Can J Sport Sci. 1990;

8. Schmidt R, Wrisberg C. Aprendizagem e performance motora: uma abordagem da aprendizagem baseada no problema. Uma abordagem da aprendizagem baseada no ....2001.

9. Balista VG. PhysioJoy Sistema de Realidade Virtual para Avaliação e Reabilitação de Déficit Motor. SBC - Proc SBGames 2013. 2013;16-20.

10. Mendes IS, Camargo CAX, Cavalheiro GL, Cardoso A, Lima FPS, Lima OM, et al. Realidade virtual: desenvolvimento e aplicabilidade no tratamento da dor secundária ao câncer de mama. 2013;4-7.12. Li Z, Zheng C, Duan C, Zhang Y, Li Q, Dou Z, et al. Rehabilitation needs of the first cohort of postacute COVID-19 patients in Hubei, China. Eur J Phys Rehabil Med. 2020;56(3):339-44.

11. Li Z, Zheng C, Duan C, Zhang Y, Li Q, Dou Z, et al. Rehabilitation needs of the first cohort of post-acute COVID-19 patients in Hubei, China. Eur J Phys Rehabil Med. 2020;56(3):339-44.

12. Boldrini P, Bernetti A, Fiore P, Bargellesi S, Bonaiuti D, Brianti R, et al. Impact of COVID-19 outbreak on rehabilitation services and Physical and Rehabilitation Medicine physicians' activities in Italy An official document of the Italian PRM Society (SIMFER). Eur J Phys Rehabil Med. 2020;56(3):315-8.

13. Lee J, Stone AJ. Combined Aerobic and Resistance Training for Cardiorespiratory Fitness, Muscle Strength, and Walking Capacity after Stroke: A Systematic Review and Meta-Analysis. J Stroke Cerebrovasc Dis [Internet]. 2020;29(1):104498. Available from: https://doi.org/10.1016/j.jstrokecerebrovasdis.2019.104498

14. Herdy A, López-Jiménez F, Terzic C, Milani M, Stein R, Carvalho T, et al. Brazilian Guidelines for cardiovascular prevention and rehabilitation. Arq Bras Cardiol. 2014;103(2)

15. Santos LST de A, Gomes E, Vilaronga J, Nunes W, Santos ACN dos, Almeida FOB de, et al. Barriers to cardiovascular rehabilitation care in a northeast city of Brazil. Acta Fisiátrica. 2017;24(2):67-71.

16. PROENÇA NL, PROENÇA NL, SPERA APN, BARCELLOS LRND, A. B. F. MAIA. Elaboração de um dispositivo mecânico estacionário promotor de rotações (cicloergômetro) para mobilização precoce, com uso de material de baixo custo. Rev UNILUS Ensino e Pesqui. 2018;55(13).

17. Adler IK. Design Thinking Design Thinking Inovação em negócios.

18. A M Anielle, Danillo Aguiar, B G L Thiag, E F G Da Alana. Cicloergômetro eletrônico de baixo custo para reabilitação cardíaca. Congresso Brasileiro de Engenharia Biomédica, 2020.

\footnotetext{
${ }^{1}$ Link para aplicativo: bit.ly/CicloCovidPrototipo1
} 